\title{
Electromagnetic Properties of Plant Leaves at Terahertz Frequencies for Health Status Monitoring
}

\author{
Adnan Zahid ${ }^{1}$, Student Member, Hasan Tahir Abbas ${ }^{2}$, Member IEEE, Hadi Heidari ${ }^{1}$, Senior Member, IEEE, Muhammad Imran ${ }^{1}$, \\ Senior Member, IEEE, Akram Alomainy ${ }^{3}$, Senior Member IEEE, Qammer H. Abbasi ${ }^{1}$, Senior Member, IEEE \\ ${ }^{1}$ School of Electronics and Nanoscale Engineering, University of Glasgow, Glasgow, U.K. \\ ${ }^{2}$ Department of Electrical and Computer Engineering, Texas A\&M University Doha Qatar \\ ${ }^{3}$ School of Electronic Engineering and Computer Science, Queen Mary University of London, London, U.K. \\ 1Email: a.zahid.1@ research.gla.ac.uk, \{Hadi.Heidari, Muhammad.Imran \& Qammer.Abbasi@glasgow.ac.uk\} \\ ${ }^{2}$ Email: Hasan.Abbas@qatar.tamu.edu, ${ }^{3}$ Email: a.alomainy@qmul.ac.uk
}

\begin{abstract}
The realization of water as an important and fundamental component requires in photosynthesis, nutritional transport, and to the effective and timely growth of plant leaves. In this work, a novel technique has been presented to determine the electromagnetic parameters of plant leaves. The measurements results of electromagnetic parameters are discussed in detail. Upon a close observation, it has been found both permittivity and a refractive index of fresh leaves showed a decline trend in their peak values from day 1 to 4 due to the rapid evaporation of moisture in tissues of leaves, and variations occurred in the morphological structure of fresh and water-stressed leaves. The technique proposed here can be employed for feasible and non-invasive plant leaves health monitoring.
\end{abstract}

Index Terms: water content, plant leaves, terahertz (THz), sensing, agriculture

\section{INTRODUCTION}

$\mathrm{O}$ ver the past decade, growing scarcity of water resources has posed a huge challenge in the plant science sector. For this purpose, many techniques [1] have been progressed at various levels to determine the reliable information of water status (WS) in plants leaves to enable cultivators and growers to make swift and effective decisions [1]. The quantitative determination of $\mathrm{WC}$ in leaves provides significant and valuable information especially in proper irrigation management and facilitates to circumvent any unforeseen situation of plant drought stress [1][2]. Therefore, evaluation of leaf's water status in leaves is of high importance and crucial to all cultivators, horticulturists, plant physiologists, and biochemists. Interestingly, water content (WC) in plant leaves is believed to have a strong absorption of Terahertz $(\mathrm{THz})$ frequency. In this regard, the applications of $\mathrm{THz}$ technology have enthralled many researchers and scientists in various disciplines of applied plant biology [2][3].

Some of the notable contributions and advancements of $\mathrm{THz}$ obtained in various fields are security imaging of imperceptible objects [4], medical imaging for non-invasive diagnostics of skin and dental care treatment [6][7], material characterization [5], high frequency communications [8], and processing the effective and quality method of controlling the food [1]. Moreover, terahertz (THz) technology have also shown distinctive advancements in exploring the molecular changes of the water content (WC) in the leaf due to its high sensitivity and penetration feature of $\mathrm{THz}$ radiation to the absorption of water by the leaves [9] as shown in Fig. 1. However, researchers are of strong view that its strong potentials to disseminate reasonably through plants is still to be discovered more in depth [1][5].
Over the past few years, the role of water and its effective distribution to plants is now indispensable given the ever-increasing rate of the aridification of land, attributed to global climate change [5] [10]. Many conventional techniques [9-13] have been suggested for the estimation of water content in leaves which proven to be highly reliable but have some limitations [11-13]. They are considered to be unsuitable for long-standing studies of some plant leaves due to their destructive nature [14-17]. Moreover, authors [15-17] suggested they are considered as time-consuming and their validity of measurements data cannot be guaranteed [15-17].

Furthermore, some non-destructive methods including thermal, infrared and hyperspectral imaging and Magnetic resonance imaging (MRI) [18] have been previously deployed to have detailed information of water status in plant leaves and made a major impact within plant society. Mostly, aforesaid approaches have been markedly suffered from some major limitations: time consuming methods, limited accessibility, and less sensitivity at cellular changes in plants and lowresolution issues [18]. Thus, a growing attention in the plant physiology has been directed to $\mathrm{THz}$ spectroscopy techniques [16-19], as a non-invasive tool for measuring leaf water status under certain conditions, such as drought stress [18]. Likewise, this too is high-cost method and its set up is of limited use [1821].

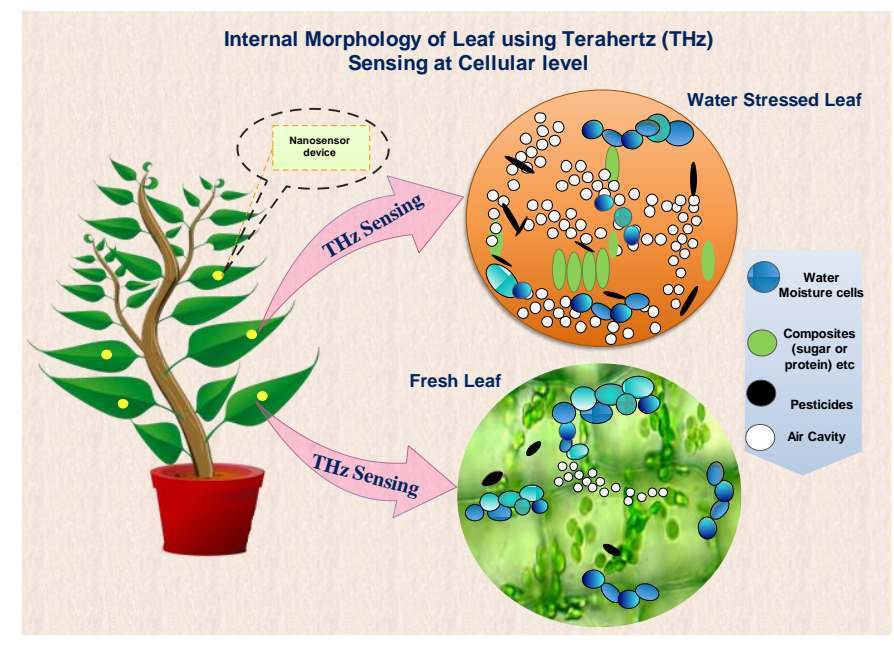

Figure 1. Internal Morphology of Fresh and Water stressed leaf using terahertz sensing. 


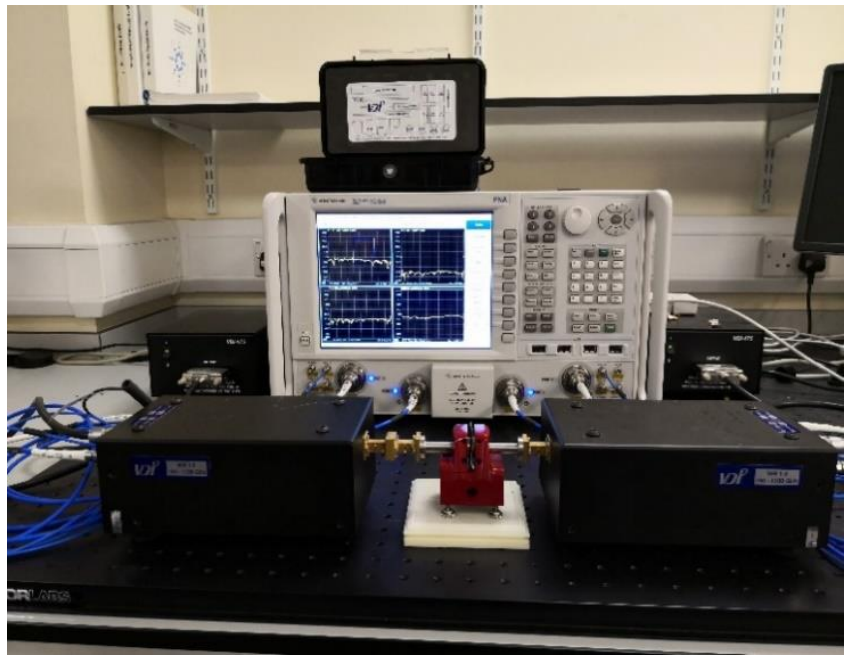

Figure 2. Experimental Setup of Swissto12 System with a frequency range from 0.75 to $1.1 \mathrm{THz}$.

This paper mainly aims to introduce a novel, simple and non-invasive method that overcome the limitations of earlier proposed methods [9-13]. In this paper, it is aimed to examine the electromagnetic parameters of leaves using the scattering (S-) parameter of a $\mathrm{THz}$ pulse. It also highlights regulations of electromagnetic parameters and refractive index variations of leaves depending on moisture content of leaves. The paper is organized as follows: Section II describes the overall methods followed by samples details. Section III illustrate the measurement results. Finally, conclusion is drawn in section IV.

\section{MATERIALS AND METHODS}

\section{A. EXPERIMENTAL SETUP}

Prior to resume measurements, it was significant to set up the THz Material Characterization Kit (MCK) supplied by Swissto12 as depicted in Fig.2, to obtain S-parameter of leaves. The system has a frequency range from 0.75 to $1.1 \mathrm{THz}$, with a thickness range from $40 \mu \mathrm{m}$ to $4 \mathrm{~mm}$. A fully two-port calibration (wR-1.0) known as Short-Open-Load-Through (SOLT) was performed to determine the measurement system's imperfections and lessen the transmission losses [2]. It includes two Polytetrafluoroethylene (PTFE) caps that are inserted at the MCK corrugated wave as shown in figure to provide uniformly compression to samples. Both the reflection (S11, S22), and transmission coefficients $\left(S_{12}, S_{21}\right)$ of samples were determined.

\section{B. SAMPLES}

Four various fresh leaves were considered for the experiment namely as; baby-leaf, basil, pea -shoot and spinach. All these leaves were detached from fresh pot and placed in laboratory under same environmental conditions for four consecutive days. These fresh plant leaves were not watered for next four days. The tested environment temperature for the measurements was $18^{\circ} \mathrm{C} \pm 0.1^{\circ} \mathrm{C}$. Three different locations on leaves were examined to observe any surface irregularities of

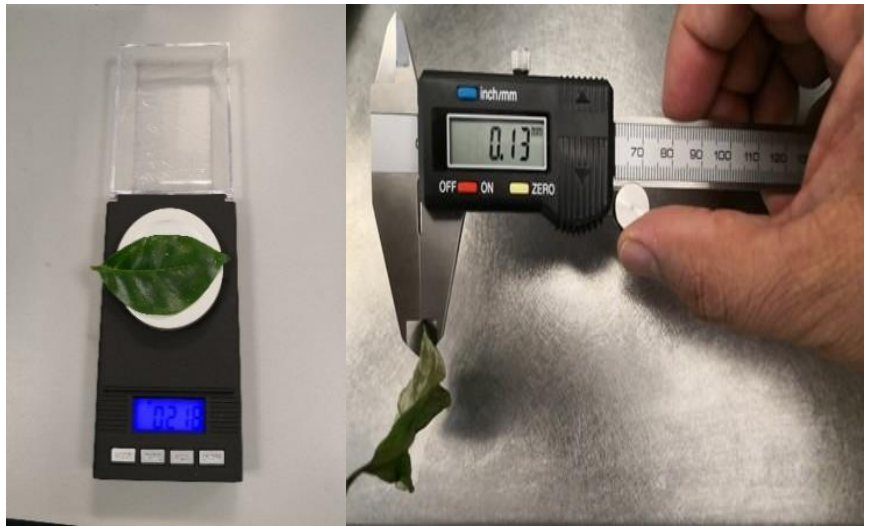

Figure 3. Demonstration of measuring the weight and thickness of leaf using Digital Scale and Vernier Calliper.

leaves and WC as shown in Fig. 4, because leaves may have different WC at various positions. The leaf's thickness and weight were also measured using Vernier calliper and digital scale respectively as shown in Fig. 3.

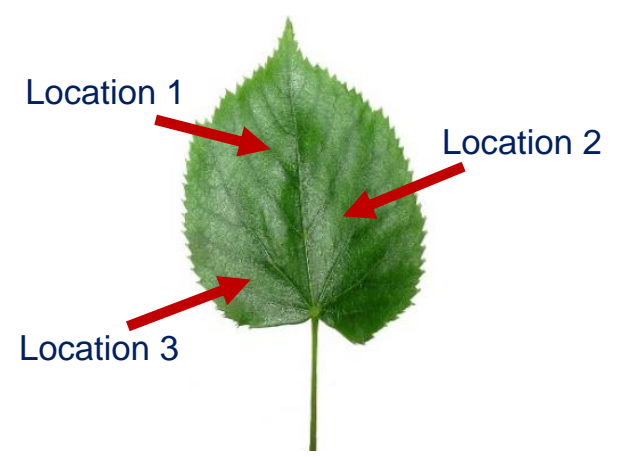

Figure 4. Three different locations considered for measuring the permittivity and refractive index response on day 1 and 4 .

\section{MEASUREMENT RESUlTS}

\section{A. PERMitTIVITY OF LEAVES ON DAY 1 AND 4}

In this study, the permittivity ' $\varepsilon_{\mathrm{r}}$ ' of all four leaves were measured for consecutive four days. Initially, transmission response $\left(S_{21}\right)$ i.e. of four leaves were obtained using network analyzer N5224A as shown in Fig. 2. It was observed that all the leaves displayed highest peak values of permittivity on day 1 when the water content in leaves were noticeably high as shown in Fig. 6(a). With passing days, it was noticed that permittivity exhibited a considerable decrement on day 4 relating to water stressed leaves. Therefore, it was deduced that fresh leaves and water stressed leaves showed significant varied response on day 1 and 4 . Furthermore, permittivity was observed at three different locations using four different orientations to observe any irregularities or structure inconsistences in leaves. However, it was observed that the differences in permittivity were negligible regardless of the location and orientation. The focus of research under this section was to establish an understanding of die-electric 

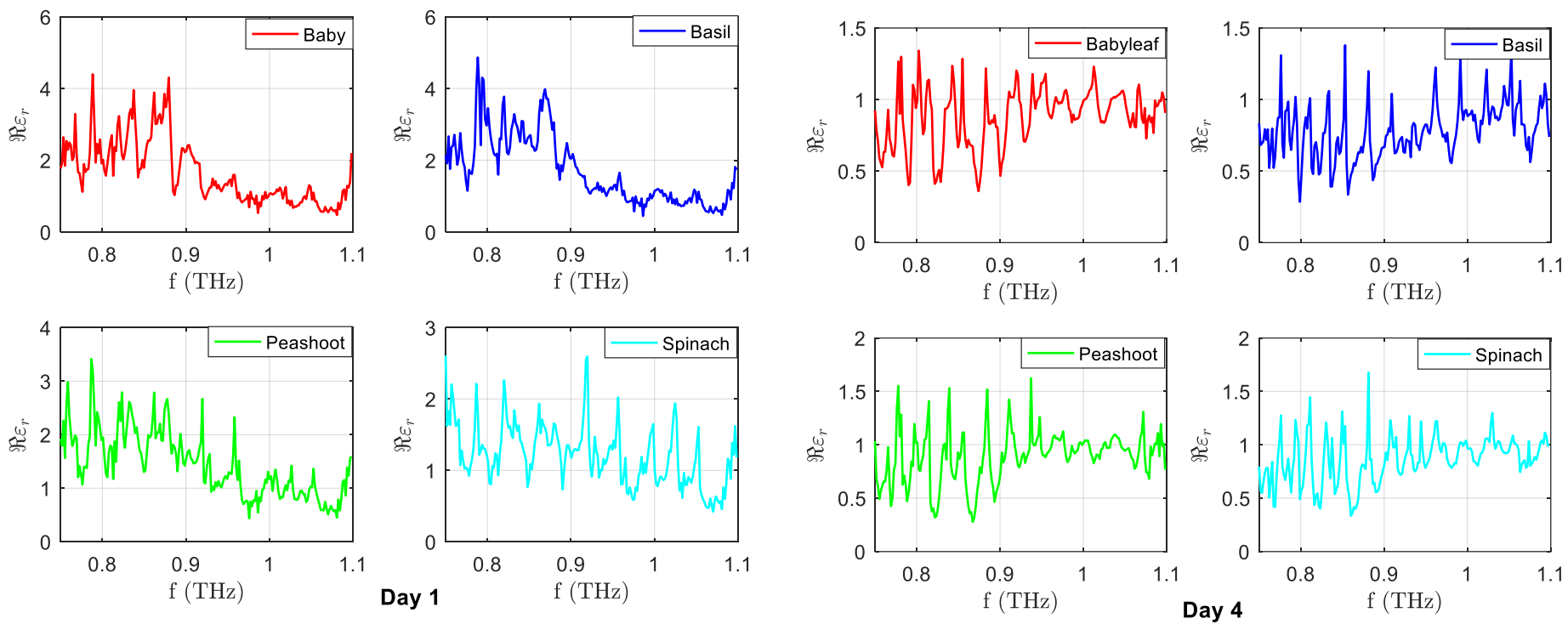

Figure 5 (a). Permittivity of all four leaves over the frequency range from 0.75 to $1.1 \mathrm{THz}$ on day 1 and 4 .
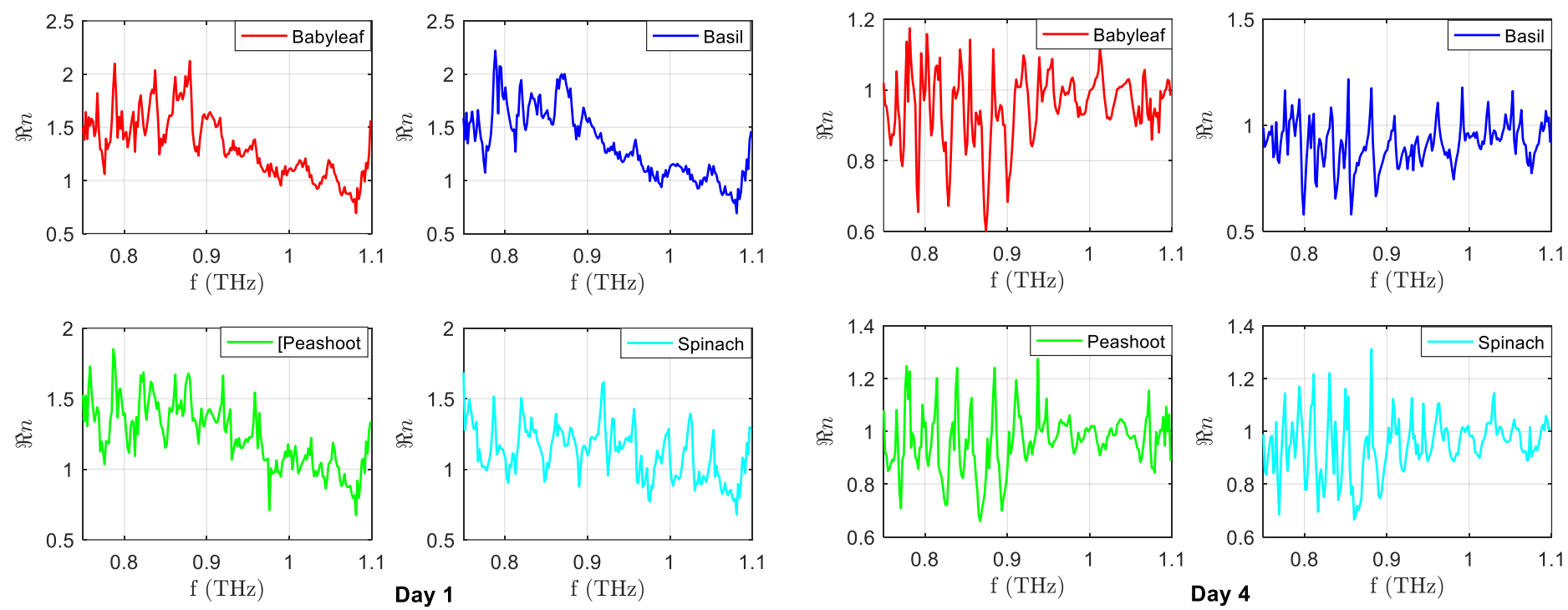

Figure 5 (b). Refractive index of all four leaves over the frequency range from 0.75 to $1.1 \mathrm{THz}$ on day 1 and 4 .

properties of various plant leaves and observed their reactions in different environmental conditions.

\section{B. REFRACTIVE INDEX OF LEAVES ON DAY 1 AND 4}

In this section, refractive index ' $n$ ' was measured for all four leaves consecutively for four days. This parameter can provide a useful information and exploit the internal characteristics of plant leaves. It was examined on day 1 that leaves exhibited individual responses depending on the presence of WC in tissue of leaves at the time of measurement. From the computed results, it was observed that with every passing day, a clear diminution response was obtained in peak values of real part of refractive index. The comparison of day 1 and 4 measurements can be seen in Fig. 6(b). Furthermore, a degree of attenuation and strong dispersion in leaves were statistically decreased with passage of time. Hence, the results identified a strong and a clear correlation of this parameter with presence of WC in leaves. Furthermore, as shown in Fig. 6(b), some leaves showed a sensitive response in the range of 0.75 to $1 \mathrm{THz}$ by showing high peaks and then gradually reduced as the frequency was increased and became transparent to $\mathrm{THz}$ waves.

\section{CONCLUSION}

This paper presented a novel approach of determining the electromagnetic parameters of plant leaves using a terahertz frequency range from 0.75 to $1.1 \mathrm{THz}$ for health status monitoring. For this purpose, electromagnetic parameters of four fresh leaves were established through the measured scattering parameters. At the same time, physical features of leaves such as weight and thickness were also determined. It was evidently observed that declining trend was transpired in 
the peak values of permittivity and refractive index from the day 1 to 4 due to the swift evaporation of moisture content in tissue of leaves. Considering the current global climate change, the proposed state-of-the-art technique is deemed to have more simple, reliable response for the overall monitoring and maintaining the health status of plant leaves.

\section{ACKNOWLEDGEMENT}

Adnan Zahid is funded by EPSRC Scholarship programme. Sincere appreciation to CSI members.

\section{REFERENCES}

[1] M. El Beyrouthya, "Nanotechnologies: Novel Solutions for Sustainable Agriculture," Adv. Crop Sci. Technol., vol. 02, no. 03, p. 8863,2014

[2] Zahid, A., Yang, K., Heidari, H. , Li, C., Imran, M. A. , Alomainy, A. and Abbasi, Q. H. (2018) Terahertz Characterisation of Living Plant Leaves for Quality of Life Assessment Applications. In: Microwave and Radar Week (MIKON-2018 and Baltic URSI Symposium-2018, Poznań, Poland, 14-17 May 2018,

[3] A. Afsharinejad, A. Davy, and M. Naftaly, "Variability of Terahertz Transmission Measured in Live Plant Leaves," IEEE Geosci. Remote Sens. Lett., vol. 14, no. 5, pp. 636-638, 2017.

[4] J. F. Federici, B. Schulkin, F. Huang, D. Gary, R. Barat, F. Oliveira, and D. Zimdars, "THz imaging and sensing for security applications, explosives, weapons and drugs," Semiconductor Science and Technology, vol. 20, no. 7, p. S266, 2005

[5] A. Afsharinejad, A. Davy, P. O'Leary, and C. Brenann, "Transmission through single and multiple layers of plant leaves at thz frequencies," in GLOBECOM 2017 - 2017 IEEE Global Communications Conference, Dec 2017, pp. 1-6.

[6] R. M. Woodward, B. E. Cole, V. P. Wallace, R. J. Pye, D. D. Arnone, E. H. Linfield, and M. Pepper, "Terahertz pulse imaging in reflection geometry of human skin cancer and skin tissue," Physics in Medicine \& Biology, vol. 47, no. 21, p. 3853, 2002.

[7] R. L. Smith, "Diagnostic modalities," Clinical Orthopaedics and Related, Research®, vol. 427, p. S174, 2004.

[8] M. Naftaly and R. E. Miles, "Terahertz time-domain spectroscopy for material characterization," Proceedings of the IEEE, vol. 95, no. 8, pp. 1658-1665, Aug 2007.

[9] N. Born et al., "Monitoring Plant Drought Stress Response Using Terahertz Time-Domain Spectroscopy," Plant Physiol., vol. 164, no. 4, pp. 1571-1577, 2014.

[10] V. Torres, I. Palacios, J. C. Iriarte, I. Liberal, L. G. Santesteban, C. Miranda, J. B. Royo, and R. Gonzalo, "Monitoring water status of grapevine by means of thz waves," Journal of Infrared, Millimeter, and Terahertz Waves, vol. 37, no. 5, pp. 507-513, May 2016.

[11] L. G. Santesteban, I. Palacios, C. Miranda, J. C. Iriarte, J. B. Royo, and R. Gonzalo, "Terahertz time domain spectroscopy allows contactless monitoring of grapevine water status," Frontiers in Plant Science, vol. 6, no. June, pp. 1-9, 2015.

[12] C. Jördens, M. Scheller, B. Breitenstein, D. Selmar, and M. Koch, "Evaluation of leaf water status by means of permittivity at terahertz frequencies," Journal of biological physics, vol. 35 , no. 3, pp. 5564, 2009.

[13] R. Gente, N. Born, A. Velauthapillai, J. C. Balzer, and M. Koch, "Monitoring the water content of plant leaves with thz time domain spectroscopy," in 2015 40th International Conference on Infrared, Millimeter, and Terahertz waves (IRMMW-THz), Aug 2015,p p. 1,2 .

[14] R. Gente and M. Koch, "Monitoring leaf water content with thz and sub-thz waves," Plant Methods, vol. 11, no. 1, p. 15, Mar 2015.

[15] N. Born, D. Behringer, S. Liepelt, S. Beyer, M. Schwerdtfeger, B. Ziegenhagen, and M. Koch, "Monitoring plant drought stress response using terahertz time-domain spectroscopy," Plant Physiology, vol. 164,no. 4, pp. 1571-1577, 2014.

[16] B. Breitenstein, M. Scheller, M. K. Shakfa, T. Kinder, T. MüllerWirts, M. Koch, and D. Selmar, "Introducing terahertz technology into plant biology: A novel method to monitor changes in leaf water status," Journal of Applied Botany and Food Quality, vol. 84, no. 2, pp. 158-161, 2011.

[17] P. Nie, F. Qu, L. Lin, T. Dong, Y. He, Y. Shao, and Y. Zhang, "Detection of water content in rapeseed leaves using terahertz spectroscopy," Sensors, vol. 17, no. 12, 2017. [Online]. Available: http://www.mdpi.com/1424-8220/17/12/2830

[18] Z. Song, S. Yan, Z. Zang, Y. Fu, D.Wei, H. L. Cui, and P. Lai, "Temporal and spatial variability of water status in plant leaves by Terahertz imaging," IEEE Transactions on Terahertz Science and Technology, vol. 8, no. 5, pp. 520-527, 2018

[19] S. C. Saha, J. P. Grant, Y. Ma, A. Khalid, F. Hong, and D. R. S. Cumming, "Terahertz frequency-domain spectroscopy method for vector characterization of liquid using an artificial dielectric," IEEE Transactions on Terahertz Science and Technology, vol. 2, no. 1, pp. 113-122, Jan 2012.

[20] S. Hadjiloucas, L. S. Karatzas, and J. W. Bowen, "Measurements of leaf water content using terahertz radiation," IEEE Transactions on Microwave Theory and Techniques, vol. 47, no. 2, pp. 142-149, 1999.

[21] U. S. de Cumis, J.-H. Xu, L. Masini, R. Degl'Innocenti, P. Pingue, F. Beltram, A. Tredicucci, M. S. Vitiello, P. A. Benedetti, H. E. Beere, and D. A. Ritchie, "Terahertz confocal microscopy with a quantum cascade laser source," Opt. Express, vol. 20, no. 20, pp. 21 924-21 931, Sep 2012. 